\title{
Symmetry Analysis, Bäcklund Transformations, and Interaction Solutions for an AB Modified KdV System
}

\author{
Man Jia (iD \\ Laboratory of Clean Energy Storage and Conversion, School of Physical Science and Technology, Ningbo University, \\ Ningbo 315211, China \\ Correspondence should be addressed to Man Jia; jiaman@nbu.edu.cn
}

Received 19 July 2019; Revised 4 November 2019; Accepted 26 November 2019; Published 17 January 2020

Academic Editor: Antonio Scarfone

Copyright (c) 2020 Man Jia. This is an open access article distributed under the Creative Commons Attribution License, which permits unrestricted use, distribution, and reproduction in any medium, provided the original work is properly cited.

An $\mathrm{AB}$ modified $\mathrm{KdV}(\mathrm{AB}-\mathrm{mKdV})$ system which can be used to describe two-place event is studied in this manuscript. Because the AB-mKdV system is considered as a special reduction of the famous AKNS system, the properties of the AKNS system are first revealed by using symmetry analysis. The nonlocal symmetries related to truncated Painlevé expansion, the finite transformation, and the symmetry reduction solutions of the AKNS system are presented. The corresponding Bäcklund transformations and the interaction solutions of the $\mathrm{AB}-\mathrm{mKdV}$ system are constructed based on the special reduction. The results demonstrate that the $\mathrm{AB}-\mathrm{mKdV}$ system possesses many kinds of interaction solutions, such as the interactions between kink and soliton and kink and cnoidal waves. The soliton can be changed from bright to dark during propagation.

\section{Introduction}

The study of symmetry is one of the most important and powerful methods in physics and mathematics because knowledge of symmetries can enhance our understanding of complicated physical phenomena, to simplify and solve problems and to deepen our standing of nature. It is particularly fundamental to find the symmetries of the integrable systems because symmetries can help to find infinitely conservation laws, generate new integrable systems, etc. Recent studies show that the nonlocal symmetry is not only related to Painlevé property, Darboux transformations, and Bäcklund transformations but can also be used to find some types of interaction solutions among different types of nonlinear excitations, such as solitons, cnoidal waves, Airy waves, and Bessel waves [1-3].

In 2013, a nonlinear nonlocal Schrödinger equation is introduced which possesses Lax pair and an infinite number of conservation laws [4] with the help of $P T$ symmetry where $x \rightarrow-x$ and $t \rightarrow-t$. Later, the AB systems, possessing shiftedparity $\left(P_{s}\right)$ and delayed time reversal $\left(T_{d}\right)$ symmetries, were introduced by Li et al. [5] which are extensions of $P T$ symmetry to describe two-place physical events. A special AB-KdV system is established to qualitatively describe two real events, the atmospheric blocking which happened in November 2007 and January 2008, respectively, while the atmospheric blockings are responsible for the heavy snow disaster in Southern China in the winter 2007/2008 [6]. Another special $\mathrm{AB}-\mathrm{KdV}$ system is constructed in [7], and the exact $P_{s} T_{d}$ invariant and $P_{s} T_{d}$ symmetric breaking solutions are found. Recent studies show that reverse space and reverse time nonlocal nonlinear integrable equations can be introduced from the remarkably simple symmetry reductions of general AKNS scattering problems [8]. Many nonlinear systems, such as KdV equation [9], mKdV equation, NLS equation and Sine-Gordon equation, $b$-family equation, and Novikov equation [10], can be extended to shifted-parity and delayed time reversal $\mathrm{AB}$ systems.

A special AB-mKdV system is

$$
\left\{\begin{array}{l}
A_{t}+A_{x x x}+6 A B A_{x}=0 \\
B=\widehat{f} A= \pm \widehat{P}_{s} \widehat{T}_{d} A= \pm A\left(-x+x_{0},-t+t_{0}\right)
\end{array}\right.
$$

with $x_{0}$ and $t_{0}$ being arbitrary constants which can be considered as a special reduction of the famous AKNS system 


$$
\left\{\begin{array}{l}
a_{t}+a_{x x x}+6 a b a_{x}=0, \\
b_{t}+b_{x x x}+6 a b b_{x}=0,
\end{array}\right.
$$

where $a \equiv a(x, t)$ and $b \equiv b(x, t)$. It is easy to see with the special reduction $a=A(x, t), b=B= \pm A\left(-x+x_{0},-t+t_{0}\right)$, the AKNS system (equation (2)) is reduced to the AB-mKdV system (equation (1)). Like the nonlocal modified KdV equation derived from the nonlinear inviscid dissipative and equivalent barotropic vorticity equation in a $\beta$-plane [11], we believe that the AB-mKdV system (equation (1)) can also be derived from the two-layer fluid system and has a physical application to describe various two-place and time-delayed correlated events.

A complex reverse space-time nonlocal complex mKdV equation and a nonlocal real reverse space-time $\mathrm{mKdV}$ equation are introduced in [8] from the remarkably simple symmetry reductions of general AKNS scattering problems. Though the AB-mKdV (equation (1)) is also generated from the AKNS system, the system is a shifted-parity and delayed time reversal system. And we believe that the ABmKdV system (equation (1)) can be considered a more general form of the nonlocal nonlinear complex and real mKdV equation.

In [12], a special case of the AB-mKdV system with $x_{0}=t_{0}=0$ is generated from the AKNS system. The exact soliton and breather solutions are constructed through inverse scattering transform that demonstrates the special PT symmetric AB-mKdV system which has some new properties, which are different from the ones of the $\mathrm{mKdV}$ equation. Furthermore, in [5], abundant new localized structures, such as the bell-ring shape soliton (bright soliton), the kink soliton (dark soliton), and the rogue wave solutions for the $\mathrm{AB}-\mathrm{mKdV}$ system (equation (1)) where $B=-A\left(-x+x_{0}\right.$, $\left.-t+t_{0}\right)$, are revealed.

However, though many kinds of exact solutions and interesting structures for the $\mathrm{AB}-\mathrm{mKdV}$ system have been found, there are still lots of problems left. For example, is it possible to find the interaction structures the AB-mKdV system possesses? How to construct the interaction solutions of the AB-mKdV system? Does the AB-mKdV system possess new interaction properties?

In [7], an AB-KdV system is studied with the help of a coupled KdV system which supplies an effective method to study the $\mathrm{AB}$ system. Motivated by this method, we start from the AKNS system (equation (2)) to reveal the properties of the AB-mKdV system (equation (1)). Here, we will use symmetry analysis that is considered as one of the most important and powerful methods.

The paper is organized as follows. In Section 2, we first present the truncated Painlevé expansion of the AKNS system, then find out the nonlocal symmetry or the residual symmetry related to the obtained truncated Painlevé expansion. To localize the nonlocal symmetry, the AKNS system is prolonged to an enlarged system. Furthermore, the symmetry reductions related to the nonlocal symmetry are studied. By using the special reduction, all the solutions of the AKNS system can be changed to those of the AB-mKdV system. In Section 3, a special Bäcklund transformation theorem of the $A B-m K d V$ system is written down according to the results of the AKNS system. It is found that the AB-mKdV system possesses many kinds of interaction solutions, such as the interaction between kink and soliton and kink and cnoidal waves.

\section{Nonlocal Symmetry of the AKNS System}

Symmetry study plays an important role in natural science, especially in physics and mathematics. Recent studies show nonlocal symmetry related to Painlevé expansion has been successfully used to find the interaction solutions of nonlinear integrable systems [13-15]. In this section, we will start from the truncated Painlevé expansion of the AKNS system.

2.1. The Truncated Painlevé Expansion. The first step to search for nonlocal symmetry of a nonlinear system is to find the truncated Painlevé expansion. By balancing the nonlinear and leading dispersive terms, it is easy to write down the truncated Painlevé expansion of the AKNS system (equation (2)) as

$$
\begin{aligned}
& a=\frac{a_{0}}{f}+a_{1}, \\
& b=\frac{b_{0}}{f}+b_{1},
\end{aligned}
$$

where $a_{i}, b_{i}, i=1,2$, and $f$ are functions of $\{x, t\}$. Substituting the expansion (equation (3)) into the AKNS system (equation (2)), we have

$$
\begin{aligned}
a_{1 t} & +a_{1 x x x}+6 a_{1} b_{1} a_{1 x} \\
& +f^{-1}\left[a_{0 t}+a_{0 x x x}+6 b_{1}\left(a_{0} a_{1}\right)_{x}+6 a_{1} b_{0} a_{1 x}\right] \\
& -f^{-2}\left[a_{0} f_{t}+a_{0} f_{x x x}+3\left(a_{0 x} f_{x}\right)_{x}+6 a_{0} a_{1} b_{1} f_{x}\right. \\
& \left.-6 a_{0 x}\left(a_{0} b_{1}+a_{1} b_{0}\right)-6 a_{0} b_{0} a_{1 x}\right]+6 f^{-3}\left[\left(a_{0} f_{x}^{2}\right)_{x}\right. \\
& \left.+a_{0 x} a_{0} b_{0}-a_{0} f_{x}\left(a_{0} b_{1}+a_{1} b_{0}\right)\right] \\
& -6 a_{0} f^{-4} f_{x}\left(a_{0} b_{0}+f_{x}^{2}\right)=0 \\
& \\
b_{1 t} & +b_{1 x x x}+6 a_{1} b_{1} b_{1 x}+f^{-1}\left[b_{0 t}+b_{0 x x x}\right. \\
& \left.+6 a_{1}\left(b_{0} b_{1}\right)_{x}+6 a_{0} b_{1} b_{1 x}\right]-f^{-2}\left[b_{0} f_{t}\right. \\
& +b_{0} f_{x x x}+3\left(b_{0} f_{x}\right)_{x}+6 b_{0} a_{1} b_{1} f_{x} \\
& \left.-6 b_{0 x}\left(a_{0} b_{1}+a_{1} b_{0}\right)-6 a_{0} b_{0} b_{1 x}\right] \\
& +6 f^{-3}\left[\left(b_{0} f_{x}^{2}\right)_{x}+a_{0} b_{0} b_{0 x}-b_{0} f_{x}\left(a_{1} b_{0}+a_{0} b_{1}\right)\right] \\
& -6 b_{0} f^{-4} f_{x}\left(a_{0} b_{0}+f_{x}^{2}\right)=0 .
\end{aligned}
$$

Vanishing the coefficients of all the powers of $f$ in equations (4) and (5), we can obtain the determining equations for $a_{i}, b_{i}, i=1,2$, and $f$.

To vanish the coefficients of $f^{-4}$ in equations (4) and (5) and link the AKNS system to the AB-mKdV system, there are only two possible cases 


$$
\begin{aligned}
& a_{0}=f_{x}, \\
& b_{0}=-f_{x}, \\
& a_{0}=i f_{x} \\
& b_{0}=i f_{x} .
\end{aligned}
$$

Case 1. For $a_{0}=f_{x}, b_{0}=-f_{x}$, vanishing all the coefficients of $f$ gives

$$
\begin{aligned}
& a_{1}=-\frac{f_{x x}}{2 f_{x}}, \\
& b_{1}=\frac{f_{x x}}{2 f_{x}},
\end{aligned}
$$

with a constraint condition

$$
f_{t}=\frac{3 f_{x x}^{2}}{2 f_{x}}-f_{x x x}
$$

which can be considered as a Schwarzian equation [16].

Thus, if $f$ is a solution of the Schwarzian equation (equation (8)), the truncated Painlevé expansion of the AKNS system is

$$
\begin{aligned}
& a=\frac{f_{x}}{f}-\frac{f_{x x}}{2 f_{x}}, \\
& b=-\frac{f_{x}}{f}+\frac{f_{x x}}{2 f_{x}} .
\end{aligned}
$$

Case 2. If $a_{0}=i f_{x}, b_{0}=i f_{x}$, one can find that

$$
\begin{aligned}
& a_{1}=-i \frac{f_{x x}}{2 f_{x}}, \\
& b_{1}=-i \frac{f_{x x}}{2 f_{x}},
\end{aligned}
$$

by vanishing all the coefficients of $f$ in equations (4) and (5) with $f$ satisfying equation (8).

In this case, the truncated Painleve expansion of the AKNS system becomes

$$
\begin{aligned}
& a=i \frac{f_{x}}{f}-i \frac{f_{x x}}{2 f_{x}}, \\
& b=i \frac{f_{x}}{f}-i \frac{f_{x x}}{2 f_{x}}
\end{aligned}
$$

where $f$ satisfies the constraint Schwarzian equation (equation (8)).

Now the truncated Painlevé expansion of the AKNS system (equation (2)) is obtained with two cases. The results show that the system may be a complex one or a real one which will lead to a complex $\mathrm{AB}-\mathrm{mKdV}$ system or a real
AB-mKdV system. Next, we will present the nonlocal symmetry of the AKNS system (equation (2)) related to the truncated Painlevé expansion.

2.2. Nonlocal Symmetry. The symmetry

$$
\sigma=\left(\begin{array}{c}
\sigma^{a} \\
\sigma^{b}
\end{array}\right)
$$

means under the infinitesimal transformation

$$
\left(\begin{array}{l}
a \\
b
\end{array}\right) \rightarrow\left(\begin{array}{l}
a \\
b
\end{array}\right)+\varepsilon\left(\begin{array}{l}
\sigma^{a} \\
\sigma^{b}
\end{array}\right)
$$

the AKNS system (equation (2)) is form invariant, where $\left\{\sigma^{a}, \sigma^{b}\right\}$ is a solution of the linearized equation of the original AKNS system:

$$
\sigma_{t}^{a}+\sigma_{x x x}^{a}+6 a_{x}\left(\sigma^{a} b+a \sigma^{b}\right)+6 a b \sigma_{x}^{a}=0
$$

$$
\sigma_{t}^{b}+\sigma_{x x x}^{b}+6 b_{x}\left(\sigma^{a} b+a \sigma^{b}\right)+6 a b \sigma^{b}=0 .
$$

It is not difficult to find that $\left\{a_{0}, b_{0}\right\}$ in the truncated Painlevé expansion (equation (3)) is a symmetry with respect to the solution $\left\{a_{1}, b_{1}\right\}$ of the AKNS system (equation (2)) which can be proved by substituting $\left\{\sigma^{a}=a_{0}, \sigma^{b}=b_{0}\right\}$ into the symmetry equation (equations (14) and (15)) with the solution $\left\{a=a_{1}, b=b_{1}\right\}$.

On the other hand, the functions $f, f_{x}$, and $f_{x x}$ are linked with $\{a, b\}$ nonlocally via equations (9) and (11), the symme$\operatorname{try}\left\{\sigma^{a}=a_{0}, \sigma^{b}=b_{0}\right\}$ is nonlocal. The nonlocal symmetry theorem related to truncated Painlevé expansion is written down.

Theorem 1. Nonlocal symmetry theorem. If $f$ is a solution of the Schwarzian equation (equation (8)), then

$$
\sigma_{1}=\left(\begin{array}{c}
\sigma^{a} \\
\sigma^{b}
\end{array}\right)=\left(\begin{array}{c}
f_{x} \\
-f_{x}
\end{array}\right)
$$

is a nonlocal symmetry of the AKNS system (equation (2)) for the solution

$$
\begin{aligned}
& a=-\frac{f_{x x}}{2 f_{x}}, \\
& b=\frac{f_{x x}}{2 f_{x}},
\end{aligned}
$$

so is

$$
\sigma_{2}=\left(\begin{array}{c}
\sigma^{a} \\
\sigma^{b}
\end{array}\right)=\left(\begin{array}{l}
i f_{x} \\
i f_{x}
\end{array}\right),
$$

for the solution 


$$
\begin{aligned}
\sigma^{a} & =-\frac{i f_{x x}}{2 f_{x}}, \\
\sigma^{b} & =-\frac{i f_{x x}}{2 f_{x}} .
\end{aligned}
$$

The theorem can be verified by directly substituting the results into the symmetry definition equation (equations (14) and (15)).

2.3. Localize the Nonlocal Symmetry. It is known that nonlocal symmetries are related to many kinds of nontrivial transformations such as the Darboux transformations (DTs), Bäcklund transformations (BTs), and Möbious transformations by localizing the nonlocal symmetries. In order to localize the nonlocal symmetry, the AKNS system (equation (2)) is prolonged to an enlarged system

$$
\begin{aligned}
a_{t}+a_{x x x}+6 a b a_{x} & =0, \\
b_{t}+b_{x x x}+6 a b b_{x} & =0, \\
f_{t} & =-f_{x x x}+\frac{3}{2} \frac{f_{x x}^{2}}{f_{x}}, \\
g & =f_{x},
\end{aligned}
$$

by introducing a new dependent variable $g=f_{x}$ with the constraint condition.

It is known that the symmetry of the enlarged system is the solution of the linearized system of equations (20), (21), (22), and (23) reading as

$$
\begin{array}{r}
\sigma_{t}^{a}+\sigma_{x x x}^{a}+6 a_{x}\left(\sigma^{a} b+a \sigma^{b}\right)+6 a b \sigma_{x}^{a}=0, \\
\sigma_{t}^{b}+\sigma_{x x x}^{b}+6 b_{x}\left(\sigma^{a} b+a \sigma^{b}\right)+6 a b \sigma_{x}^{b}=0, \\
\sigma_{t}^{f} f_{x}+\sigma_{x x x}^{f} f_{x}-3 \sigma_{x x}^{f} f_{x x}+f_{x x x} \sigma_{x}^{f}+f_{t} \sigma_{x}^{f}=0, \\
\sigma^{g}-\sigma_{x}^{f}=0 .
\end{array}
$$

For the enlarged system (equations (20), (21), (22), and (23)), it is easy to verify the Schwarzian equation (equation (22)) possesses a famous symmetry $\sigma^{f}=-f^{2}+c_{1} f+c_{0}$ satisfying equation (26), so we can directly derive that the symmetry of the prolonged system (equations (20), (21), (22), and (23)) has the form of

$$
\left(\begin{array}{c}
\sigma^{a} \\
\sigma^{b} \\
\sigma^{f} \\
\sigma^{g}
\end{array}\right)=\left(\begin{array}{c}
g \\
-g \\
-f^{2}+c_{1} f+c_{0} \\
-2 f g+c_{1} g
\end{array}\right),
$$

$$
\left(\begin{array}{c}
\sigma^{a} \\
\sigma^{b} \\
\sigma^{f} \\
\sigma^{g}
\end{array}\right)=\left(\begin{array}{c}
i g \\
i g \\
-f^{2}+c_{1} f+c_{0} \\
-2 f g+c_{1} g
\end{array}\right),
$$

where $c_{0}$ and $c_{1}$ are arbitrary constants.

Thus, the nonlocal symmetries (equations (16) and (18)) are localized and become Lie point symmetries (equations (28) and (29)) for the prolonged system (equations (20), (21), (22), and (23)) by introducing the function $g$.

2.4. Initial Problem of the Prolonged System. The last step of the standard symmetry analysis method is solving the initial problem. Using the initial problem and localized symmetry, we can find finite transformation and the related symmetry reductions of the prolonged system.

The initial value problem is

$$
\begin{aligned}
& \frac{d a^{\prime}(\varepsilon)}{d \varepsilon}=g^{\prime}(\varepsilon), \quad a^{\prime}(0)=a, \\
& \frac{d b^{\prime}(\varepsilon)}{d \varepsilon}=-g^{\prime}(\varepsilon), \quad b^{\prime}(0)=b, \\
& \frac{d f^{\prime}(\varepsilon)}{d \varepsilon}=-f^{\prime}(\varepsilon)^{2}+c_{1} f^{\prime}(\varepsilon)+c_{0}, \quad f^{\prime}(0)=f, \\
& \frac{d g^{\prime}(\varepsilon)}{d \varepsilon}=-2 f^{\prime}(\varepsilon) g^{\prime}(\varepsilon)+c_{1} g^{\prime}(\varepsilon), \quad g^{\prime}(0)=g,
\end{aligned}
$$

for the first kind of symmetry (equation (28)) and

$$
\begin{aligned}
& \frac{d a^{\prime}(\varepsilon)}{d \varepsilon}=i g^{\prime}(\varepsilon), \quad a^{\prime}(0)=a, \\
& \frac{d b^{\prime}(\varepsilon)}{d \varepsilon}=i g^{\prime}(\varepsilon), \quad b^{\prime}(0)=b, \\
& \frac{d f^{\prime}(\varepsilon)}{d \varepsilon}=-f^{\prime}(\varepsilon)^{2}+c_{1} f^{\prime}(\varepsilon)+c_{0}, \quad f^{\prime}(0)=f, \\
& \frac{d g^{\prime}(\varepsilon)}{d \varepsilon}=-2 f^{\prime}(\varepsilon) g^{\prime}(\varepsilon)+c_{1} g^{\prime}(\varepsilon), \quad g^{\prime}(0)=g,
\end{aligned}
$$

for the second kind of symmetry (equation (29)). Solving the initial value problems, one can directly derive the finite transformation theorems.

Theorem 2. Finite transformation theorem for the symmetry (equation (28)). If $\{a, b, f, g\}$ is a solution of the enlarged system (equations (20), (21), (22), and (23)), so is $\left\{a^{\prime}, b^{\prime}, f^{\prime}, g^{\prime}\right\}$ 


$$
\begin{aligned}
a^{\prime}= & a+\frac{g\left(2 f-c_{1}\right)}{2\left(f^{2}-c_{1} f-c_{0}\right)}-\frac{\alpha g}{2\left(f^{2}-c_{1} f-c_{0}\right)} \tanh \\
& \cdot\left[\operatorname{arctanh}\left(\frac{2 f-c_{1}}{\alpha}\right)+\frac{\varepsilon \alpha}{2}\right], \\
b^{\prime}= & b-\frac{g\left(2 f-c_{1}\right)}{2\left(f^{2}-c_{1} f-c_{0}\right)}+\frac{\alpha g}{2\left(f^{2}-c_{1} f-c_{0}\right)} \tanh \\
& \cdot\left[\operatorname{arctanh}\left(\frac{2 f-c_{1}}{\alpha}\right)+\frac{\varepsilon \alpha}{2}\right], \\
f^{\prime}= & \frac{\alpha}{2} \tanh \left[\operatorname{arctanh}\left(\frac{2 f-c_{1}}{\alpha}\right)+\frac{\varepsilon \alpha}{2}\right]+\frac{c_{1}}{2}, \\
g^{\prime}= & -\frac{\alpha^{2} g}{4\left(f^{2}-c_{1} f-c_{0}\right)} \operatorname{sech}^{2} \\
& \cdot\left[\operatorname{arctanh}\left(\frac{2 f-c_{1}}{\alpha}\right)+\frac{\varepsilon \alpha}{2}\right],
\end{aligned}
$$

for $\alpha \equiv \sqrt{c_{1}^{2}+4 c_{0}} \neq 0$, and $\left\{a^{\prime \prime}, b^{\prime \prime}, f^{\prime \prime}, g^{\prime \prime}\right\}$

$$
\begin{aligned}
& a^{\prime \prime}=a+\frac{2 \varepsilon g}{2 \varepsilon f-c_{1} \varepsilon+2}, \\
& b^{\prime \prime}=b-\frac{2 \varepsilon g}{2 \varepsilon f-c_{1} \varepsilon+2}, \\
& f^{\prime \prime}=\frac{2 c_{1} \varepsilon f-c_{1}^{2} \varepsilon+4 f}{2\left(2 \varepsilon f-c_{1} \varepsilon+2\right)}, \\
& g^{\prime \prime}=\frac{4 g}{\left(2 \varepsilon f-c_{1} \varepsilon+2\right)^{2}},
\end{aligned}
$$

for $\alpha=0$ with $c_{0}$ and $c_{1}$ being two arbitrary constants.

Theorem 3. Finite transformation theorem for the symmetry (equation (29)). If $\{a, b, f, g\}$ is a solution of the prolonged system (equations (20), (21), (22), and (23)), then $\left\{a^{\prime}, b^{\prime}, f^{\prime}, g^{\prime}\right\}$

$$
\begin{aligned}
& a^{\prime}=a+\frac{2 i g \tanh (\varepsilon \alpha / 2)}{\left(2 f-c_{1}\right) \tanh (\varepsilon \alpha / 2)-\alpha}, \\
& b^{\prime}=b+\frac{2 i g \tanh (\varepsilon \alpha / 2)}{\left(2 f-c_{1}\right) \tanh (\varepsilon \alpha / 2)-\alpha} \\
& f^{\prime}=\frac{\alpha}{2} \tanh \left[\operatorname{arctanh}\left(\frac{2 f-c_{1}}{\alpha}\right)+\frac{\varepsilon \alpha}{2}\right]+\frac{c_{1}}{2} \\
& g^{\prime}=-\frac{\alpha^{2} g}{4\left(f^{2}-c_{1} f-c_{0}\right)} \sec h^{2}\left[\operatorname{arctanh}\left(\frac{2 f-c_{1}}{\alpha}\right)+\frac{\varepsilon \alpha}{2}\right]
\end{aligned}
$$

for $\alpha \neq 0$, and $\left\{a^{\prime \prime}, b^{\prime \prime}, f^{\prime \prime}, g^{\prime \prime}\right\}$

$$
\begin{aligned}
a^{\prime \prime} & =a+\frac{2 i \varepsilon g}{\varepsilon\left(2 f-c_{1}\right)-2}, \\
b^{\prime \prime} & =b+\frac{2 i \varepsilon g}{\varepsilon\left(2 f-c_{1}\right)-2} \\
f^{\prime \prime} & =\frac{2 c_{1} \varepsilon f-c_{1}^{2} \varepsilon+4 f}{2\left(2 \varepsilon f-c_{1} \varepsilon+2\right)} \\
g^{\prime \prime} & =\frac{4 g}{\left(2 \varepsilon f-c_{1} \varepsilon+2\right)^{2}}
\end{aligned}
$$

are also solutions of the prolonged system.

According to these theorems, starting from any seed solutions of the enlarged system (equations (20), (21), (22), and (23)), infinitely many new solutions, especially interaction solutions among different nonlinear excitations, can be obtained.

2.5. Lie Point Symmetry of the Prolonged System. Now that the nonlocal symmetry of the enlarged system is localized, it is natural to find the classical Lie point symmetry to search for more new reductions. The famous first fundamental theorem of Lie $[17,18]$ is a standard method used widely to find Lie point symmetry algebras. In this section, we use the standard method to obtain the Lie point symmetry and symmetry reductions of the prolonged system. For simplicity, we present the symmetry reductions related to the symmetry (equation (28)) for the prolonged system (equations (20), (21), (22), and (23)).

According to the classical Lie group theory [17], the enlarged system (equations (20), (21), (22), and (23)) possesses the Lie point symmetry

$$
\begin{aligned}
\left(\begin{array}{c}
\sigma^{a} \\
\sigma^{b} \\
\sigma^{f} \\
\sigma^{g}
\end{array}\right)= & X(x, t, a, b, f, g)\left(\begin{array}{c}
a_{x} \\
b_{x} \\
f_{x} \\
g_{x}
\end{array}\right)+T(x, t, a, b, f, g)\left(\begin{array}{l}
a_{t} \\
b_{t} \\
f_{t} \\
g_{t}
\end{array}\right) \\
& -\left(\begin{array}{c}
A_{1}(x, t, a, b, f, g) \\
B_{1}(x, t, a, b, f, g) \\
F(x, t, a, b, f, g) \\
G(x, t, a, b, f, g)
\end{array}\right),
\end{aligned}
$$

where $\left\{\sigma^{a}, \sigma^{b}, \sigma^{f}, \sigma^{g}\right\}$ is a solution of the linearized equation of the enlarged system which means the enlarged system is invariant under the infinitesimal transformation 


$$
\begin{aligned}
a \rightarrow & +\varepsilon\left[A_{1}(x, t, a, b, f, g)-X(x, t, a, b, f, g) a_{x}\right. \\
& \left.-T(x, t, a, b, f, g) a_{t}\right] \equiv a+\varepsilon \sigma^{a}, \\
b \rightarrow & b+\varepsilon\left[B_{1}(x, t, a, b, f, g)-X(x, t, a, b, f, g) b_{x}\right. \\
& \left.-T(x, t, a, b, f, g) b_{t}\right] \equiv b+\varepsilon \sigma^{b}, \\
f \rightarrow & f+\varepsilon\left[F(x, t, a, b, f, g)-X(x, t, a, b, f, g) f_{x}\right. \\
& \left.-T(x, t, a, b, f, g) f_{t}\right] \equiv f+\varepsilon \sigma^{f}, \\
g \rightarrow & g+\varepsilon\left[G(x, t, a, b, f, g)-X(x, t, a, b, f, g) g_{x}\right. \\
& \left.-T(x, t, a, b, f, g) g_{t}\right] \equiv a+\varepsilon \sigma^{g} .
\end{aligned}
$$

Substituting equation (36) into the linearized equation of the enlarged system (equations (24), (25), (26), and (27)) and eliminating $a_{t}, b_{t}$, and $f_{x}$ by the original system, then collecting the coefficients of $a, b, f$, and $g$ and their derivatives, an overdetermined set of equations for the unknown functions $X, T, A_{1}, B_{1}, F$, and $G$ are obtained with the solution

$$
\begin{aligned}
X & =c_{1} x+c_{2}, \\
T & =3 c_{1} t+c_{3}, \\
F & =c_{4} f^{2}-c_{5} f-c_{6}, \\
G & =2 c_{4} f g-c_{1} g-c_{5} g, \\
A_{1} & =-c_{1} a-c_{4} g, \\
B_{1} & =-c_{1} b+c_{4} g,
\end{aligned}
$$

where $c_{i}, i=1, \cdots, 6$, are arbitrary constants.

Once the Lie point symmetry is given, we can use it to find the symmetry reductions of the enlarged system. By solving the characteristic equation

$$
\begin{aligned}
\frac{d x}{X(x, t, a, b, f, g)} & =\frac{d t}{T(x, t, a, b, f, g)}=\frac{d a}{A_{1}(x, t, a, b, f, g)} \\
& =\frac{d b}{B_{1}(x, t, a, b, f, g)}=\frac{d f}{F(x, t, a, b, f, g)} \\
& =\frac{d g}{G(x, t, a, b, f, g)},
\end{aligned}
$$

the group invariants and the reduction solutions are presented in 5 cases.

Case $1\left(c_{1}=0, c_{2} \neq 0, c_{3} \neq 0, c_{4} \neq 0\right)$. The prolonged system (equations (20), (21), (22), and (23)) possesses a solution

$$
\begin{aligned}
& a=\frac{c_{3}^{2} F_{\xi \xi}}{2 c_{2}\left(c_{3} F_{\xi}-c_{2}\right)}-\frac{\alpha_{1}\left(c_{3} F_{\xi}-c_{2}\right)}{2 c_{2}^{2}} \tanh \left[\frac{\alpha_{1}}{2 c_{2}}(F+x)\right], \\
& b=-\frac{c_{3}^{2} F_{\xi \xi}}{2 c_{2}\left(c_{3} F_{\xi}-c_{2}\right)}+\frac{\alpha_{1}\left(c_{3} F_{\xi}-c_{2}\right)}{2 c_{2}^{2}} \tanh \left[\frac{\alpha_{1}}{2 c_{2}}(F+x)\right],
\end{aligned}
$$

$$
\begin{aligned}
& f=-\frac{\alpha_{1}}{2 c_{4}} \tanh \left[\frac{\alpha_{1}}{2 c_{2}}(F+x)\right]+\frac{c_{5}}{2 c_{4}}, \\
& g=\frac{\alpha_{1}^{2}\left(c_{3} F_{\xi}-c_{2}\right)}{2 c_{2}^{2} c_{4}\left\{\cosh \left[\left(\alpha_{1} / c_{2}\right)(F+x)\right]+1\right\}},
\end{aligned}
$$

where $F$ satisfies the reduction equation

$$
\begin{aligned}
F_{\xi \xi \xi}= & \frac{3 c_{3} F_{\xi \xi}^{2}}{2\left(c_{3} F_{\xi}-c_{2}\right)}+\frac{\alpha_{1}^{2}\left(c_{3} F_{\xi}-c_{2}\right)^{3}}{2 c_{2}^{2} c_{3}^{3}} \\
& +\frac{c_{2}^{3}\left(c_{3} F_{\xi}-c_{2}\right)}{c_{3}^{4}}+\frac{c_{2}^{4}}{c_{3}^{4}},
\end{aligned}
$$

with $\alpha_{1} \equiv \sqrt{4 c_{4} c_{6}+c_{5}^{2}}$, and

$$
\xi=-\frac{c_{3}}{c_{2}} x+t
$$

Case $2\left(c_{1}=0, c_{2} \neq 0, c_{3} \neq 0, c_{4}=0, c_{5} \neq 0\right)$. In this case, if $F$ satisfies the reduction equation

$$
\begin{aligned}
F_{\xi \xi \xi}= & \frac{3\left(c_{3} F_{\xi \xi}+c_{5} F_{\xi}\right)^{2}}{2 c_{3}\left(c_{3} F_{\xi}+c_{5} F\right)}+\frac{\left(2 c_{2}^{3}+3 c_{3} c_{5}^{2}\right)\left(c_{3} F_{\xi}+c_{5} F\right)}{2 c_{3}^{4}} \\
& -\frac{c_{5}\left(c_{2}^{3}+c_{3} c_{5}^{2}\right) F}{c_{3}^{4}},
\end{aligned}
$$

then

$$
\begin{aligned}
& f=e^{-c_{5} x / c_{2}} F-\frac{c_{6}}{c_{5}}, \\
& g=e^{-c_{5} x / c_{2}} \frac{c_{5} F+c_{3} F_{\xi}}{c_{2}}, \\
& a=\frac{c_{3}^{2} F_{\xi \xi}+2 c_{3} c_{5} F_{\xi}+c_{5}^{2} F}{2 c_{2}\left(c_{3} F_{\xi}+c_{5} F\right)}, \\
& b=-\frac{c_{3}^{2} F_{\xi \xi}+2 c_{3} c_{5} F_{\xi}+c_{5}^{2} F}{2 c_{2}\left(c_{3} F_{\xi}+c_{5} F\right)},
\end{aligned}
$$

with the invariant variable

$$
\xi=-\frac{c_{3}}{c_{2}} x+t
$$

is a solution of the prolonged system (equations (20), (21), (22), and (23)).

Case $3\left(c_{1}=0, c_{2} \neq 0, c_{3} \neq 0, c_{4}=0, c_{5}=0\right)$. If $F$ is a solution of the reduction equation

$$
F_{\xi \xi \xi}=\frac{3 c_{3}^{4} F_{\xi \xi}^{2}+2 c_{2}^{3} c_{3} F_{\xi}^{2}+2 c_{2}^{3} c_{6} F_{\xi}}{2 c_{3}^{3}\left(c_{3} F_{\xi}+c_{6}\right)}
$$


where

$$
\xi=\frac{-c_{3} x+c_{2} t}{c_{2}},
$$

then

$$
\begin{aligned}
& f=\frac{c_{6} x}{c_{2}}+F, \\
& g=-\frac{c_{3} F_{\xi}+c_{6}}{c_{2}}, \\
& a=\frac{c_{3}^{2} F_{\xi \xi}}{2 c_{2}\left(c_{3} F_{\xi}+c_{6}\right)}, \\
& b=-\frac{c_{3}^{2} F_{\xi \xi}}{2 c_{2}\left(c_{3} F_{\xi}+c_{6}\right)},
\end{aligned}
$$

is a solution of the prolonged system (equations (20), (21), (22), and (23)).

Case $4\left(c_{1} \neq 0, c_{2} \neq 0, c_{3} \neq 0, c_{4} \neq 0\right)$. In this case, the similarity solution can be written as

$$
\begin{aligned}
f= & -\frac{\alpha_{1} \tanh \left[\left(\alpha_{1} F / 2\right)+\left(\alpha_{1} / 2 c_{1}\right) \ln \left(c_{1} x+c_{2}\right)\right]-c_{5}}{2 c_{4}}, \\
g= & \frac{\alpha_{1}^{2}\left(3 c_{1} \xi F_{\xi}-1\right)}{2 c_{4}\left(c_{1} x+c_{2}\right)\left\{\cosh \left[\left(\alpha_{1} F / 2\right)+\left(\alpha_{1} / 2 c_{1}\right) \ln \left(c_{1} x+c_{2}\right)\right]+1\right\}}, \\
a= & \frac{3 c_{1}^{2} \xi\left(\xi F_{\xi \xi}+F_{\xi}\right)-\alpha_{1}\left(3 c_{1} \xi F_{\xi}-1\right)^{2}+c_{1}\left(3 c_{1} \xi F_{\xi}-1\right)}{2\left(c_{1} x+c_{2}\right)\left(3 c_{1} \xi F_{\xi}-1\right)} \\
& -\frac{\alpha_{1}\left(3 c_{1} \xi F_{\xi}-1\right)}{e^{\alpha_{1} F}\left(c_{1} x+c_{2}\right)^{\alpha_{1} / c_{1}}+1}, \\
b= & -\frac{3 c_{1}^{2} \xi\left(\xi F_{\xi \xi}+F_{\xi}\right)-\alpha_{1}\left(3 c_{1} \xi F_{\xi}-1\right)^{2}+c_{1}\left(3 c_{1} \xi F_{\xi}-1\right)}{2\left(c_{1} x+c_{2}\right)\left(3 c_{1} \xi F_{\xi}-1\right)} \\
& +\frac{\alpha_{1}\left(3 c_{1} \xi F_{\xi}-1\right)}{e^{\alpha_{1} F}\left(c_{1} x+c_{2}\right)^{\alpha_{1} / c_{1}}+1},
\end{aligned}
$$

with similarity variable

$$
\xi=\frac{3 c_{1} t+c_{3}}{3 c_{1}\left(c_{1} x+c_{2}\right)^{3}},
$$

where the group invariant function $F$ is determined by the reduction equation

$$
\begin{aligned}
F_{\xi \xi \xi}= & \frac{9 c_{1}\left(\xi F_{\xi \xi}+F_{\xi}\right)^{2}}{2 \xi\left(3 c_{1} \xi F_{\xi}-1\right)}-\frac{3\left(\xi F_{\xi \xi}+F_{\xi}\right)}{\xi^{2}}+\frac{\alpha_{1}^{2}\left(3 c_{1} \xi F_{\xi}-1\right)^{3}}{c_{1}^{3} \xi^{3}} \\
& +\frac{\left(105 c_{1}^{3} \xi+2\right)\left(3 c_{1} \xi F_{\xi}-1\right)}{c_{1}^{4} \xi^{4}}+\frac{54 c_{1}^{3} \xi+1}{81 c_{1}^{4} \xi^{4}} .
\end{aligned}
$$

Case $5\left(c_{1} \neq 0, c_{2} \neq 0, c_{3} \neq 0, c_{4}=0, c_{5} \neq 0\right)$. If $F$ is a solution of the reduction equation

$$
\begin{aligned}
F_{\xi \xi \xi}= & \frac{\left(3 c_{1} \xi F_{\xi \xi}+3 c_{1} F_{\xi}+c_{5} F_{\xi}\right)^{2}}{2 c_{1} \xi\left(3 c_{1} \xi F_{\xi}+c_{5} F\right)} \\
& -\frac{3 c_{1} \xi F_{\xi \xi}+3 c_{1} F_{\xi}+c_{5} F_{\xi}}{c_{1} \xi^{2}} \\
& +\frac{\left(105 c_{1}^{3} \xi+54 c_{1}^{2} c_{5} \xi+9 c_{1} c_{5}^{2} \xi+2\right) F_{\xi}}{54 c_{1}^{3} \xi^{3}} \\
& -\frac{c_{5}\left(c_{1}^{2}-c_{5}^{2}\right) F}{54 c_{1}^{3} \xi^{3}}
\end{aligned}
$$

then

$$
\begin{aligned}
& f=\left(c_{1} x+c_{2}\right)^{-c_{5} / c_{1}} F-\frac{c_{6}}{c_{5}}, \\
& g=-\left(c_{1} x+c_{2}\right)^{-\left(c_{1}+c_{5}\right) / c_{1}}\left(c_{5} F+3 c_{1} \xi F_{\xi}\right), \\
& a=\frac{9 c_{1}^{2} \xi^{2}+6 c_{1}\left(2 c_{1}+c_{5}\right) \xi F_{\xi}+c_{5}\left(c_{1}+c_{5}\right) F}{2\left(c_{1} x+c_{2}\right)\left(3 c_{1} \xi F_{\xi}+c_{5} F\right)}, \\
& b=-\frac{9 c_{1}^{2} \xi^{2}+6 c_{1}\left(2 c_{1}+c_{5}\right) \xi F_{\xi}+c_{5}\left(c_{1}+c_{5}\right) F}{2\left(c_{1} x+c_{2}\right)\left(3 c_{1} \xi F_{\xi}+c_{5} F\right)},
\end{aligned}
$$

with the invariant

$$
\xi=\frac{3 c_{1} t+c_{3}}{3 c_{1}\left(c_{1} x+c_{2}\right)^{3}}
$$

is a solution of the prolonged system (equations (20), (21), (22), and (23)).

According to the above results, many kinds of solutions of the prolonged system (equations (20), (21), (22), and (23)) can be found out by symmetry reductions with localized symmetries. The reduction solutions exhibit the prolonged system (equations (20), (21), (22), and (23)) which possesses rich structures. Here, we do not show it in detail.

\section{Bäcklund Transformation and Interaction Solutions of the AB-mKdV System}

Due to the special relation between the AKNS system and the AB-mKdV system, we can change some solutions of the AKNS system to those of the AB-mKdV system. For example, with the special reduction $a=A, b=B= \pm A\left(-x+x_{0}\right.$, $\left.-t+t_{0}\right)$, the AKNS system will be reduced to the AB$\mathrm{mKdV}$ system. Thus, we obtain some special Bäcklund transformation and interaction solutions of the AB-mKdV system according to the known results of the AKNS system.

3.1. Bäcklund Transformation Related to Nonlocal Symmetry. From the finite transformation theorem of the AKNS system, it is direct and easy to construct the Bäcklund transformation theorem for the AB-mKdV system (equation (1)). 
Theorem 4. Bäcklund transformation theorem. If $A$ is a solution of the $A B-m K d V$ system

$$
\left\{\begin{array}{l}
A_{t}+A_{x x x}+6 A B A_{x}=0, \\
B=\widehat{f} A=-\widehat{P}_{s} \widehat{T}_{d} A=-A\left(-x+x_{0},-t+t_{0}\right),
\end{array}\right.
$$

and $f$ is a solution of the Schwarzian equation (equation (8)), then

$$
\begin{aligned}
A^{\prime}(\varepsilon)=A & +\frac{f_{x}\left(2 f-c_{1}\right)}{2\left(f^{2}-c_{1} f-c_{0}\right)} \\
& -\frac{\alpha f_{x}}{2\left(f^{2}-c_{1} f-c_{0}\right)} \tanh \left[\operatorname{arctanh}\left(\frac{2 f-c_{1}}{\alpha}\right)+\frac{\varepsilon \alpha}{2}\right], \\
A^{\prime \prime}(\varepsilon)=A & +\frac{2 \varepsilon f_{x}}{2 \varepsilon f-c_{1} \varepsilon+2}
\end{aligned}
$$

are also solutions of the $A B-m K d V$ system (equation (58)) with $\alpha \equiv \sqrt{c_{1}^{2}+4 c_{0}} \neq 0$ and $c_{1}, c_{0}$ being arbitrary constants.

Theorem 5. Bäcklund transformation theorem. If $f$ is a solution of the Schwarzian equation (equation (8)) and A satisfies the AB-mKdV system

$$
\left\{\begin{array}{l}
A_{t}+A_{x x x}+6 A B A_{x}=0, \\
B=\widehat{f} A=\widehat{P}_{s} \widehat{T}_{d} A=A\left(-x+x_{0},-t+t_{0}\right),
\end{array}\right.
$$

then

$$
A^{\prime}=A+\frac{2 i f_{x} \tanh (\varepsilon \alpha / 2)}{\left(2 f-c_{1}\right) \tanh (\varepsilon \alpha / 2)-\alpha},
$$

for $\alpha \neq 0$, and

$$
A^{\prime \prime}=A+\frac{2 i \varepsilon g}{\varepsilon\left(2 f-c_{1}\right)-2}
$$

are also solutions of the $A B-m K d V$ system (equation (60)).

We can verify the theorems by directly substituting the solutions into the $\mathrm{AB}-\mathrm{mKdV}$ system with $f$ satisfying the Schwarzian equation (equation (8)), respectively.

3.2. Exact Solutions for the AB-mKdV System. Because the $\mathrm{AB}-\mathrm{mKdV}$ system is related to the AKNS system by the special reduction, all the exact solutions of the AB-mKdV system can be obtained from those of the AKNS system. Here, we list some interaction solutions of the AB-mKdV system that derived from the symmetry reduction solutions of the AKNS system.

The reduction equation (equation (44)) in Case 1 is equivalent to

$$
F_{1 \xi \xi}=\frac{3}{2} \frac{F_{1 \xi}^{2}}{F_{1}}+\frac{\alpha_{1}}{2 c_{2}^{2} c_{3}^{2}} F_{1}^{3}+\frac{c_{2}^{3}}{c_{3}^{3}} F_{1}+\frac{c_{2}^{4}}{c_{3}^{3}},
$$

by introducing $F_{1}$

$$
F_{1}=c_{3} F_{\xi}-c_{2} .
$$

It is known that the solution of equation (63) can be written as the function of the Jacobi elliptic functions which is assumed to be

$$
F_{1}=\beta_{1} \xi+\beta_{2} E_{\pi}\left[m_{0} \operatorname{sn}\left(\beta_{0} \xi, m\right), n, v\right],
$$

where $\beta_{0}, \beta_{1}, \beta_{2}, m_{0}, m$, and $n$ are constants; $\operatorname{sn}\left(\beta_{0} \xi, m\right) \equiv S_{n}$, $c n\left(\beta_{0} \xi, m\right) \equiv C_{n}$, and $d n\left(\beta_{0} \xi, m\right) \equiv D_{n}$ are the usual Jacobi elliptic functions; and $E_{\pi}(\eta, n, v)$ is the third type of incomplete elliptic integral defined by

$$
E_{\pi}(\eta, n, v)=\int_{0}^{\eta} \frac{d t}{\left(1-n t^{2}\right) \sqrt{\left(1-t^{2}\right)\left(1-v^{2} t^{2}\right)}} .
$$

Substituting equation (65) into equation (63), we can find three cases of important solutions: (1) $m_{0}=1, v=m$; (2) $m_{0}=m, n=0, v=1$; (3) $m_{0}=\sqrt{m}, n=0, v=1$.

Case $1\left(m_{0}=1, v=m\right)$. In this case, substituting equation (65) with $m_{0}=1, v=m$ into equation (63) leads to a long equation related to different powers of $S_{n}$. Vanishing all the coefficients of different powers of $S_{n}$, a unique nontrivial solution is found

$$
\begin{aligned}
& \beta_{1}=0, \\
& \beta_{2}=\frac{c_{2}\left(m^{2} n-3 m^{2}+n\right)}{a \beta_{0} m^{2}}, \\
& \alpha_{1}=-\frac{8 c_{2}^{3} m^{4}\left(m^{2} n-m^{2}-n^{2}+n\right)}{c_{3}\left(m^{2} n-3 m^{2}+n\right)^{3}}, \\
& c_{2}^{3}=\frac{2 \beta_{0} c_{3}^{3}\left(m^{2} n-3 m^{2}+n\right)}{n},
\end{aligned}
$$

with $\beta_{0}, m, n$, and $c_{3}$ being four arbitrary constants. Thus, a special solution for $F$ is found by substituting all the known results into the $F$ expression, say

$$
F=\frac{c_{2}}{c_{3}} \xi+\frac{c_{2}\left(m^{2} n-3 m^{2}+n\right)}{2 c_{3} \beta_{0} m^{2}} E_{\pi}\left[\operatorname{sn}\left(\beta_{0} \xi, m\right), n, m\right] .
$$

The corresponding solution $a$ and $b$ for the AKNS system is constructed by equations (40), (41), (42), and (43) which is

$$
\begin{aligned}
a= & \frac{\alpha_{1}\left(m^{2} n-3 m^{2}+n\right)}{c_{2} m^{2}\left(n S_{n}^{2}-1\right)} \tanh \\
& \cdot\left[\frac{c_{2}\left(m^{2} n-3 m^{2}+n\right) E_{\pi}\left(S_{n}, n, m\right)+2 \beta_{0} m^{2} c_{2} t}{4 c_{2} c_{3} \beta_{0} m^{2}}\right] \\
& +\frac{n c_{3} \beta_{0} S_{n} C_{n} D_{n}}{c_{2}\left(1-n S_{n}^{2}\right)}, \\
b= & -a .
\end{aligned}
$$




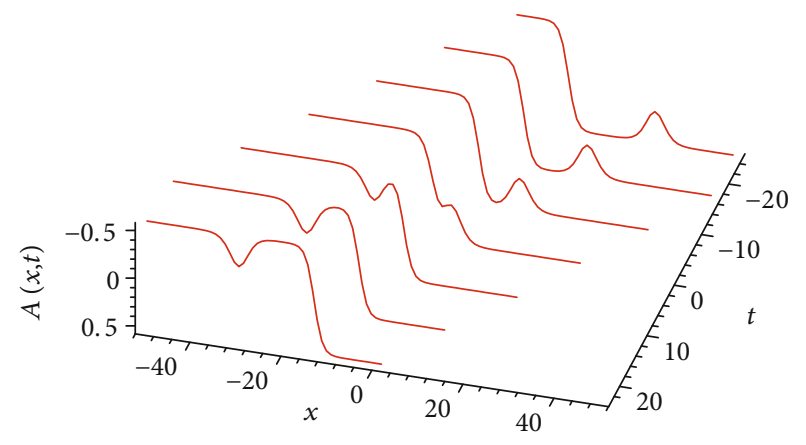

(a)

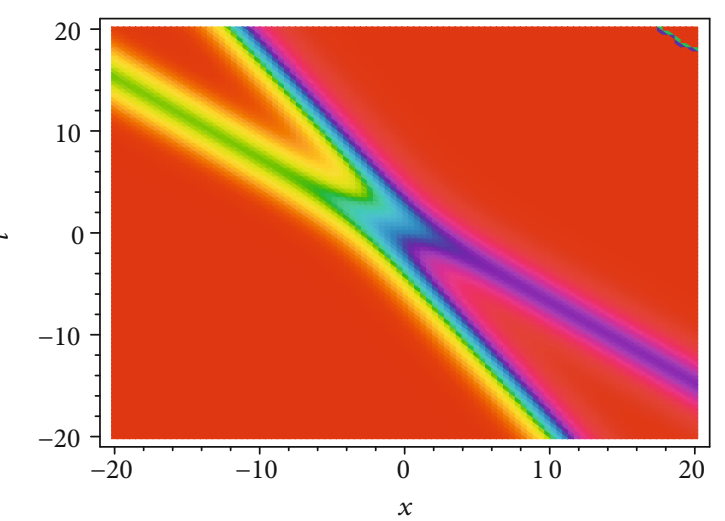

(b)

Figure 1: An interaction structure between soliton and kink for solution $A$ in equations (70) and (72) is shown in (a) with the density plot in (b) where the parameters are selected as equation (72).

Using the special reduction, we can find that the solution for the AB-mKdV system (equation (58)) is derived directly from equation (69) reading as

$$
\begin{aligned}
A= & \frac{\alpha_{1}\left(m^{2} n-3 m^{2}+n\right)}{c_{2} m^{2}\left(n{S^{\prime}}_{n}^{2}-1\right)} \tanh \\
& \cdot\left[\frac{c_{2}\left(m^{2} n-3 m^{2}+n\right) E_{\pi}\left(S_{n}^{\prime}, n, m\right)+2 \beta_{0} m^{2} c_{2}\left(t-t_{0} / 2\right)}{4 c_{2} c_{3} \beta_{0} m^{2}}\right] \\
& +\frac{n c_{3} \beta_{0} S_{n}^{\prime} C_{n}^{\prime} D^{\prime}{ }_{n}}{c_{2}\left(1-n S^{\prime 2}\right)}, \\
B= & -A\left(-x+x_{0},-t+t_{0}\right)
\end{aligned}
$$

where $S_{n}^{\prime} \equiv \operatorname{sn}\left(\beta_{0} \xi^{\prime}, m\right), C_{n}^{\prime} \equiv c n\left(\beta_{0} \xi^{\prime}, m\right), D_{n}^{\prime} \equiv d n\left(\beta_{0} \xi^{\prime}, m\right)$, and

$$
\xi^{\prime}=-\frac{c_{3}}{c_{2}}\left(x-\frac{x_{0}}{2}\right)+\left(t-\frac{t_{0}}{2}\right) .
$$

The solution may be singularity at some areas, but for the special case $m=1$, it exhibits the interactions between soliton and kink. Figure 1 shows the interaction solution between soliton and kink for $m=1$ with other parameters being selected as

$$
\begin{aligned}
t_{0} & =2, \\
x_{0} & =-2, \\
\beta_{0} & =\frac{1}{2}, \\
c_{3} & =-1, \\
n & =\frac{1}{2},
\end{aligned}
$$

in equation (70) for $A$. Figure 1(a) shows the interaction structure between soliton and kink, and Figure 1(b) shows the corresponding density plot. It is found that the soliton moves along the kink with the soliton remaining its shape. The interesting thing is during the interaction between kink and soliton, the soliton is transformed from dark to light which may be used in nonlinear optical systems. Similarly, in [19], it is pointed that bell-shaped and kinkshaped waves propagate together in description of dynamic rearrangements in biatomic lattices. But the difference is the bell-shaped localized moving defect may arise or decay in a lattice due to the propagation of a macrostrain wave.

Case $2\left(m_{0}=m, n=0, v=1\right)$. In this case, equation (65) is reduced to

$$
F_{1}=\beta_{1} \xi_{1}+\beta_{2} \operatorname{arctanh}\left[m s n\left(\beta_{0} \xi_{1}, m\right)\right] .
$$

Substituting the expression (equation (73)) into the equivalent equation (equation (63)) yields further constraints of the constants

$$
\begin{aligned}
& \beta_{1}=\frac{c_{2}\left(\mathrm{Sm}^{2}-5\right)}{4\left(1-m^{2}\right)}, \\
& \beta_{2}=\frac{c_{2}^{3}\left(m^{2}-5\right)}{4 \beta_{0} c_{3}\left(1-m^{2}\right)}, \\
& \alpha_{1}=\frac{32 c_{2}^{2}\left(1-m^{2}\right)^{2}}{\left(m^{2}-5\right)^{3}}, \\
& c_{2}^{3}=\frac{1}{2} \beta_{0}^{2} c_{3}^{3}\left(m^{2}-5\right) .
\end{aligned}
$$

Thus, the corresponding solution for the AKNS system (equation (2)) is 


$$
\begin{aligned}
a= & \frac{\alpha_{1}\left(5-m^{2}\right)\left(1+m^{2}+2 m C_{n} D_{n}-2 m^{2} S_{n}^{2}\right)}{8 c_{2}\left(1-m^{2}\right)\left(m^{2} S_{n}^{2}-m C_{n} D_{n}-1\right)} \tanh \\
& \cdot\left[\frac{\alpha_{1}\left(m^{2}-5\right) x}{8 c_{2}\left(m^{2}-1\right)}+\frac{\alpha_{1}\left(3 m^{2}+1\right) t}{8 c_{3}\left(m^{2}-1\right)}\right. \\
& \left.-\frac{\alpha_{1}\left(m^{2}-5\right) \operatorname{arctanh}\left(m S_{n}\right)}{8 \beta_{0} c_{3}\left(m^{2}-1\right)}\right] \\
& +\frac{\left(1-m^{2}\right) m \beta_{0} c_{3} S_{n}}{2 c_{2}\left(m^{2} S_{n}^{2}-m C_{n} D_{n}-1\right)}, \\
b= & -a,
\end{aligned}
$$

and the interaction solution of the $\mathrm{AB}-\mathrm{mKdV}$ system (equation (58)) is obtained

$$
\begin{aligned}
A= & \frac{\alpha_{1}\left(5-m^{2}\right)\left(1+m^{2}+2 m C^{\prime}{ }_{n}^{\prime}{ }_{n}-2 m^{2} S_{n}^{\prime 2}\right)}{8 c_{2}\left(1-m^{2}\right)\left(m^{2} S_{n^{\prime}}^{2}-m C_{n}{ }^{\prime} D_{n}^{\prime}-1\right)} \tanh \\
& \cdot\left[\frac{\alpha_{1}\left(m^{2}-5\right)}{8 c_{2}\left(m^{2}-1\right)}\left(x-\frac{x_{0}}{2}\right)+\frac{\alpha_{1}\left(3 m^{2}+1\right)}{8 c_{3}\left(m^{2}-1\right)}\left(t-\frac{t_{0}}{2}\right)\right. \\
& \left.-\frac{\alpha_{1}\left(m^{2}-5\right) \operatorname{arctanh}\left(m S^{\prime}\right)}{8 \beta_{0} c_{3}\left(m^{2}-1\right)}\right] \\
& +\frac{\left(1-m^{2}\right) m \beta_{0} c_{3} S_{n}^{\prime}}{2 c_{2}\left(m^{2} S^{\prime 2}-m C_{n}^{\prime} D_{n}^{\prime}{ }_{n}-1\right)}, \\
B= & -A\left(-x+x_{0},-t+t_{0}\right) .
\end{aligned}
$$

Solution in equation (76) is a special soliton-cnoidal wave interaction solution. Figure 2 shows the special solution for

$$
\begin{aligned}
& \beta_{0}=\frac{1}{2}, \\
& c_{3}=-1, \\
& m=\frac{1}{2}, \\
& t_{0}=2, \\
& x_{0}=-2,
\end{aligned}
$$

with Figure 2(a) being the wave interaction structure and Figure 2(b) being the density plot.

Case $3\left(m_{0}^{2}=m, n=0, v=1\right)$. The constraint condition for the constants is

$$
\begin{aligned}
& \beta_{1}=-\frac{5 c_{2} m^{2}+6 c_{2} m+5 c_{2}}{4 m^{2}-8 m+4}, \\
& \beta_{2}=-\frac{5 c_{2}\left(m^{2}+6 m+5\right)}{2 \beta_{0}\left(m^{3}-m^{2}-m+1\right)},
\end{aligned}
$$

$$
\begin{aligned}
\alpha_{1} & =-\frac{32 c_{3}^{2}(m+1)^{2}(m-1)^{4}}{c_{3}\left(5 m^{2}+6 m+5\right)^{3}}, \\
c_{2}^{3} & =-\frac{1}{2} \beta_{0}^{2} c_{3}^{3}\left(5 m^{2}+6 m+5\right),
\end{aligned}
$$

which yields a solution of the AKNS system

$$
\begin{aligned}
a= & \frac{\alpha_{1}\left(5 m^{2}+6 m+5\right)}{8(m+1)(m-1)^{2}\left(m_{0} S_{n}-1\right)} \\
& \cdot\left[m^{2}\left(m^{2}+6 m+1\right) S_{n}^{4}+4 m m_{0}(m+1) D_{n} C_{n} S_{n}^{2}\right. \\
& -2 m\left(3 m^{2}+2 m+3\right) S_{n}^{2}-4 m_{0}(m+1) C_{n} D_{n} \\
& \left.+m^{2}+6 m+1\right] \times \tanh \left[\frac{\alpha_{1}\left(5 m^{2}+6 m+5\right) x}{8 c_{2}(m-1)^{2}}\right. \\
& -\frac{\alpha_{1}\left(m^{2}+14 m+1\right) t}{8 c_{2}(m-1)^{2}} \\
& \left.+\frac{\alpha_{1}\left(5 m^{2}+6 m+5\right) \operatorname{arctanh}\left(m_{0} S_{n}\right)}{4 c_{3} \beta_{0}(m+1)(m-1)^{2}}\right] \\
& +\frac{c_{3} \beta_{0} m_{0}\left(m_{0}-1\right)^{2}\left(1+m S_{n}^{2}\right) S_{n}}{c_{2}\left(m S_{n}^{2}-1\right)\left(m^{2} S_{n}^{2}+m S_{n}^{2}+2 m_{0} C_{n} D_{n}-m-1\right)}, \\
b= & -a,
\end{aligned}
$$

with the corresponding solution of the $\mathrm{AB}-\mathrm{mKdV}$ system (equation (58))

$$
\begin{aligned}
& A=\frac{\alpha_{1}\left(5 m^{2}+6 m+5\right)}{8(m+1)(m-1)^{2}\left(m_{0} s_{n}^{\prime}-1\right)}\left[m^{2}\left(m^{2}+6 m+1\right) s_{n}^{\prime^{4}}\right. \\
& +4 m m_{0}(m+1) D_{n}^{\prime} C_{n}^{\prime} S_{n}^{\prime 2}-2 m\left(3 m^{2}+2 m+3\right) S_{n}^{2} \\
& \left.-4 m_{0}(m+1) C_{n}^{\prime} D_{n}^{\prime}+m^{2}+6 m+1\right] \\
& \times \tanh \left[\frac{\alpha_{1}\left(5 m^{2}+6 m+5\right)}{8 c_{2}(m-1)^{2}}\left(x-\frac{x_{0}}{2}\right)\right. \\
& -\frac{\alpha_{1}\left(m^{2}+14 m+1\right)}{8 c_{2}(m-1)^{2}}\left(t-\frac{t_{0}}{2}\right) \\
& \left.+\frac{\alpha_{1}\left(5 m^{2}+6 m+5\right) \operatorname{arctanh}\left(m_{0} S_{n}^{\prime}\right)}{4 c_{3} \beta_{0}(m+1)(m-1)^{2}}\right] \\
& +\frac{c_{3} \beta_{0} m_{0}\left(m_{0}-1\right)^{2}\left(1+m{S^{\prime 2}}_{n}\right) S_{n}^{\prime}}{c_{2}\left(m{S^{\prime 2}}_{n}^{2}-1\right)\left(m^{2}{S^{\prime 2}}_{n}^{2}+m{S^{\prime 2}}_{n}^{2}+2 m_{0} C_{n}^{\prime} D_{n}^{\prime}-m-1\right)}, \\
& B=-A\left(-x+x_{0},-t+t_{0}\right) \text {, }
\end{aligned}
$$

by using the reduction $A=a, B=-a\left(-x+x_{0},-t+t_{0}\right)$. The solution (equation (80)) denotes an interaction between 


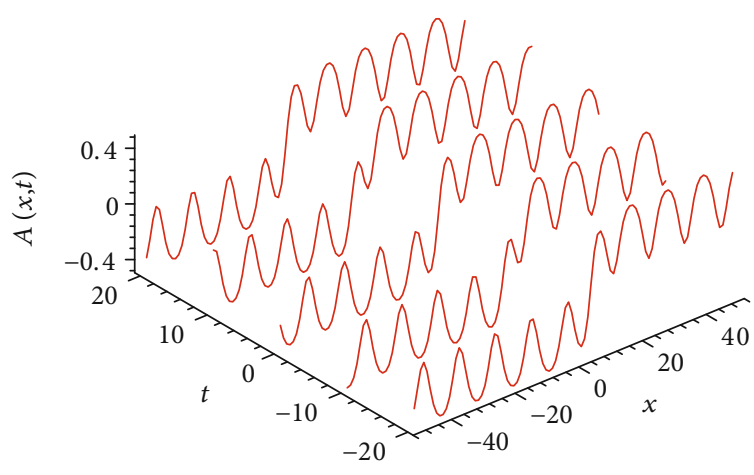

(a)

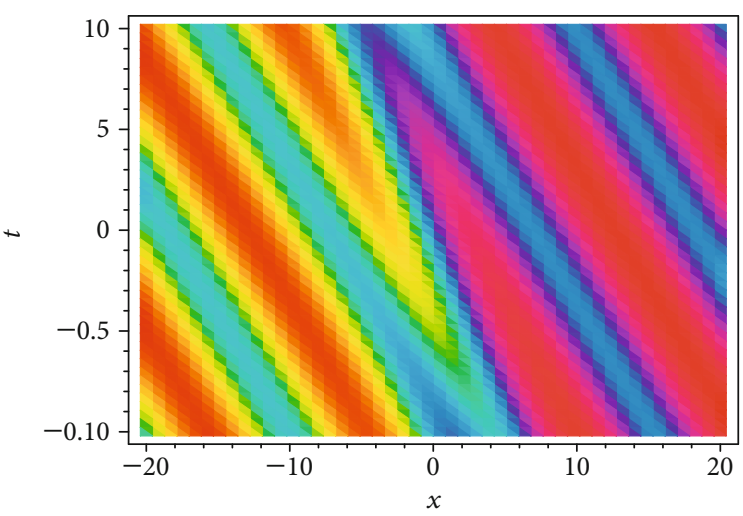

(b)

Figure 2: The interaction between kink and cnoidal wave solution for $A$ in equation (76) is shown with the parameter selected as equation (77). (a) is the interaction structure and (b) is the corresponding density plot.

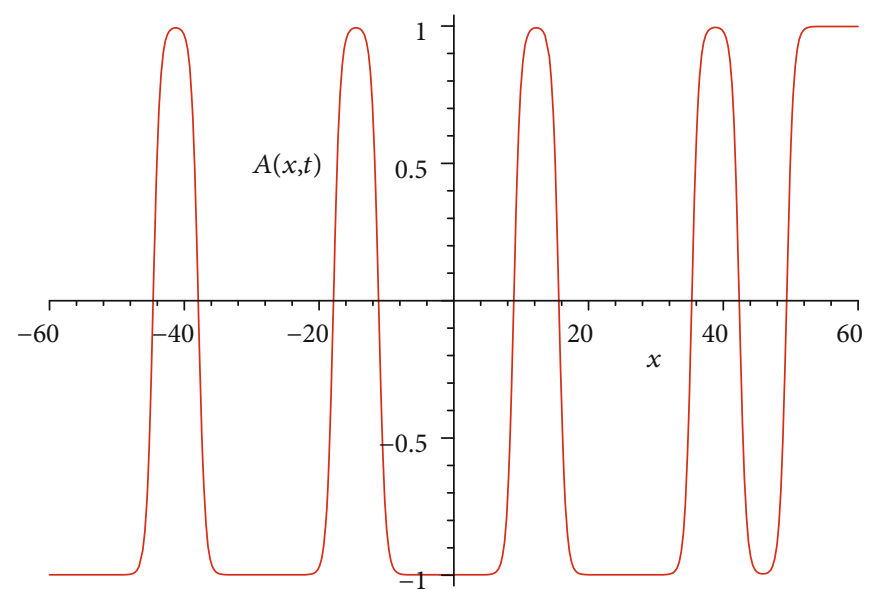

(a)

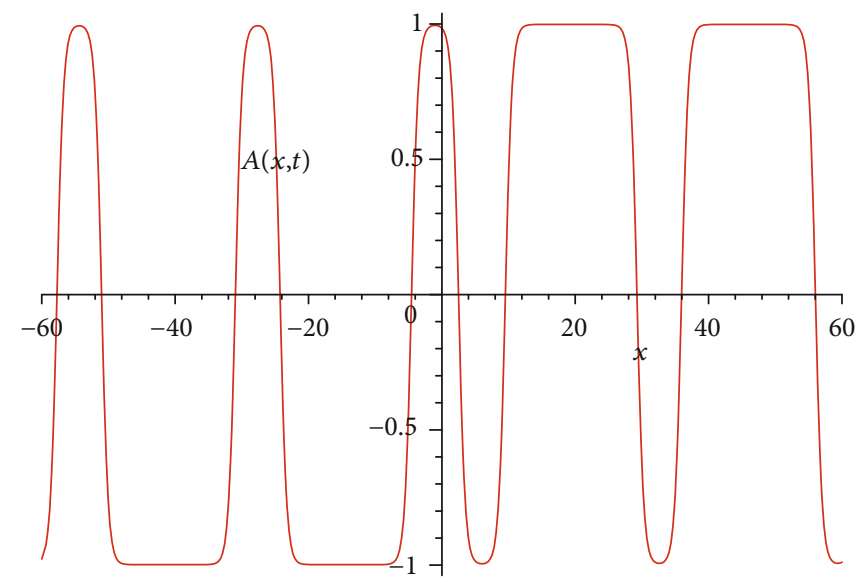

(c)

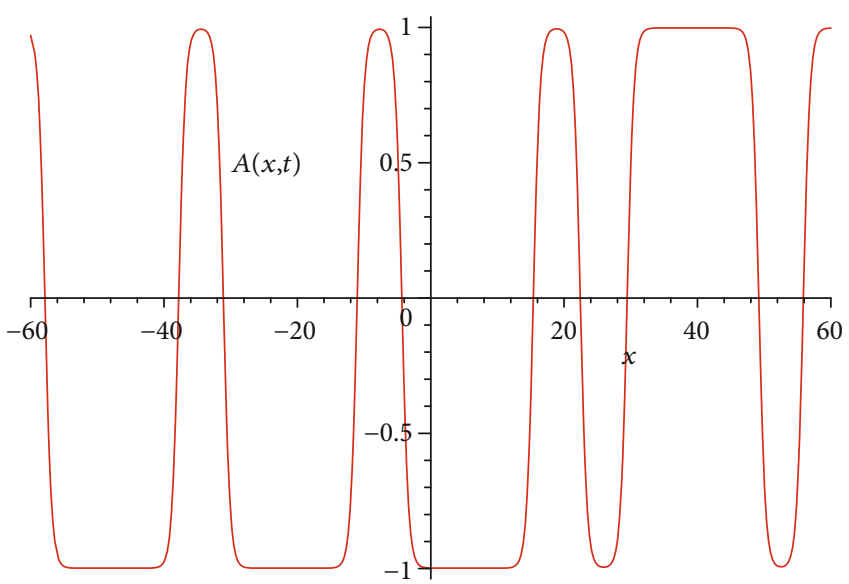

(b)

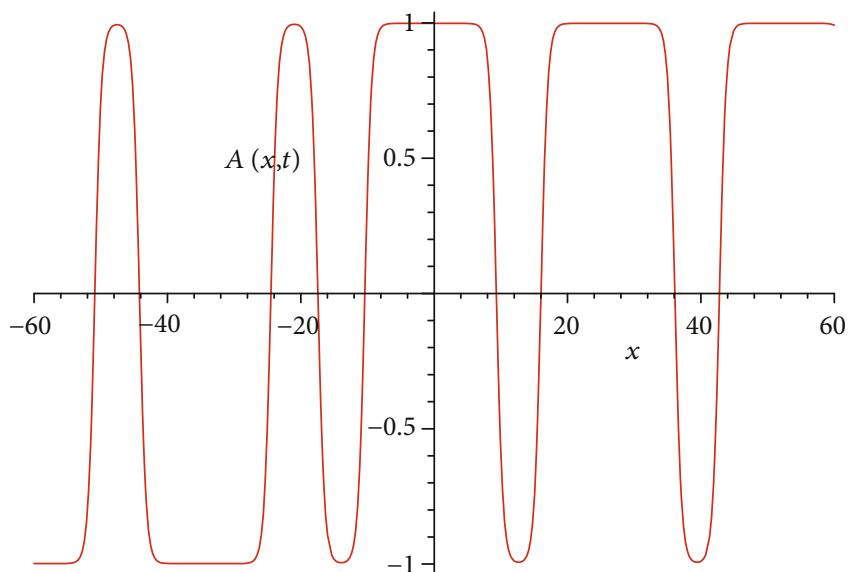

(d)

Figure 3: A special interaction solution $A$ in equation (80) with the parameters being selected as equation (81) which exhibits the interaction between soliton and cnoidal wave at (a) $t=-20$, (b) $t=-10$, (c) $t=0$, and (d) $t=10$, respectively. 
soliton and cnoidal wave. Figure 3 shows the interaction with the parameters selected as

$$
\begin{aligned}
& \beta_{0}=1, \\
& c_{3}=1, \\
& m=\frac{99}{100}, \\
& x_{0}=1, \\
& t_{0}=2,
\end{aligned}
$$

at $t=-20$ (Figure 3(a)), $t=-10$ (Figure 3(b)), $t=0$ (Figure 3(c)), and $t=10$ (Figure 3(d)).

\section{Summary and Discussion}

In summary, starting from the AKNS system (equation (2)), an $\mathrm{AB}-\mathrm{mKdV}$ system used to describe two-place events is studied. By using the nonlocal symmetry analysis, we find the Lie point symmetries and symmetry reductions of the ANKS system. Because the AB-mKdV system can be obtained by using special reduction from the AKNS system, all the conclusions of the AKNS system can be applied to those of the AB-mKdV system. The Bäcklund transformation of the $\mathrm{AB}-\mathrm{mKdV}$ system is constructed for two cases from those of the AKNS system. And the exact solutions, including $P_{s} T_{d}$ symmetric and anti- $P_{s} T_{d}$ symmetric of the $\mathrm{AB}-\mathrm{mKdV}$ system (equation (58)) are shown for simplicity. The former means that the two correlated events happen similar to each other at a different place and time, while the latter tells that they are totally different.

Studies show that the AB-mKdV system possesses rich and interesting structure solutions, especially the interaction solution structures between solitons and cnoidal waves, such as the interaction between soliton and kink and the interaction between kink and cnoidal waves. Though the interaction solution between kink and cnoidal waves has been reported in many other manuscripts, the interaction between soliton and kink has seldom been reported. The most interesting thing is that during the interaction, the soliton becomes dark from bright with the kink remaining invariant.

Though the AB-mKdV system is introduced in a mathematical manner, we believe the nonlocal $\mathrm{mKdV}$ equation can also be derived from two-layer fluid systems. More about the system will be discussed in the future.

\section{Data Availability}

No data is available.

\section{Conflicts of Interest}

The author declares that he has no conflicts of interest.

\section{Acknowledgments}

The author is indebted to Profs. S. Y. Lou and B. F. Feng for their helpful discussions. The work is supported by NNSFChina (No. 11675084) and Ningbo Natural Science Founda- tion (No. 2015A610159). The author is sponsored by K. C. Wong Magna Fund in Ningbo University.

\section{References}

[1] S. Y. Lou and X. B. Hu, "Infinitely many Lax pairs and symmetry constraints of the KP equation," Journal of Mathematical Physics, vol. 38, no. 12, pp. 6401-6427, 1997.

[2] S. Y. Lou and X. B. Hu, "Non-local symmetries via Darboux transformations," Journal of Physics A: Mathematical and General, vol. 30, no. 5, pp. L95-L100, 1997.

[3] X. R. Hu, S. Y. Lou, and Y. Chen, "Explicit solutions from eigenfunction symmetry of the Korteweg-de Vries equation," Physical Review E, vol. 85, no. 5, p. 056607, 2012.

[4] M. J. Ablowitz and Z. H. Musslimani, "Integrable nonlocal nonlinear Schrödinger equation," Physical Review Letters, vol. 110, no. 6, p. 064105, 2013.

[5] C. Li, S. Y. Lou, and M. Jia, "Coherent structure of Alice-Bob modified Korteweg de-Vries equation," Nonlinear Dynamics, vol. 93, no. 4, pp. 1799-1808, 2018.

[6] S. Y. Lou and F. Huang, "Alice-Bob physics: coherent solutions of nonlocal KdV systems," Scientific Reports, vol. 7, no. 1, p. 869, 2017.

[7] M. Jia and S. Y. Lou, "Exact PT invariant and PT symmetric breaking solutions, symmetry reductions and Bäcklund transformations for an AB-KdV system," Physics Letters A, vol. 382, no. 17, pp. 1157-1166, 2018.

[8] M. J. Ablowitz and Z. H. Musslimani, "Integrable nonlocal nonlinear equations," Studies in Applied Mathematics, vol. 139, no. 1, pp. 7-59, 2017.

[9] S. Y. Lou, 2016, ArXiv e-prints (Preprint 1603.03975).

[10] J. Wang, N. Xiong, and B. Li, "Peakon solutions of Alice-Bob $b$-family equation and Novikov equation," Advances in Mathematical Physics, vol. 2019, Article ID 1519305, p. 8, 2019.

[11] X. Y. Tang, Z. F. Liang, and X. Z. Hao, "Communications in nonlinear science and numerical simulation," Communications in Nonlinear Science and Numerical Simulation, vol. 60, pp. 62-71, 2018.

[12] J. L. Ji and Z. N. Zhu, "Soliton solutions of an integrable nonlocal modified Korteweg-de Vries equation through inverse scattering transform," Journal of Mathematical Analysis and Applications, vol. 453, no. 2, pp. 973-984, 2017.

[13] X. N. Gao, S. Y. Lou, and X. Y. Tang, "Bosonization, singularity analysis, nonlocal symmetry reductions and exact solutions of supersymmetric KdV equation," Journal of High Energy Physics, vol. 2013, article 6064, no. 5, 29 pages, 2013.

[14] C. L. Chen and S. Y. Lou, "CTE solvability, nonlocal symmetries and exact solutions of dispersive water wave system," Communications in Theoretical Physics, vol. 61, no. 5, pp. 545-550, 2014.

[15] Y. Jin, M. Jia, and S. Y. Lou, "Nonlocalization of nonlocal symmetry and symmetry reductions of the Burgers equation," Communications in Theoretical Physics, vol. 58, no. 6, pp. 795799, 2012.

[16] S. Y. Lou, "Consistent Riccati expansion for integrable systems," Studies in Applied Mathematics, vol. 134, no. 3, pp. 372-402, 2015.

[17] P. J. Olver, Applications of Lie Groups to Differential Equations, Springer New York, 1993. 
[18] G. W. Bluman and S. C. Anco, Symmetry and Integration Methods for Differential Equations, Springer, New York, 2002.

[19] A. V. Porubov and B. R. Andrievsky, "Kink and solitary waves may propagate together," Physical Review E, vol. 85, no. 4, p. 046604, 2012. 


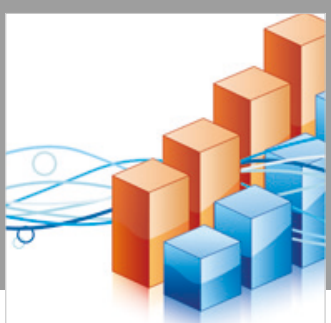

Advances in

Operations Research

\section{-n-m}
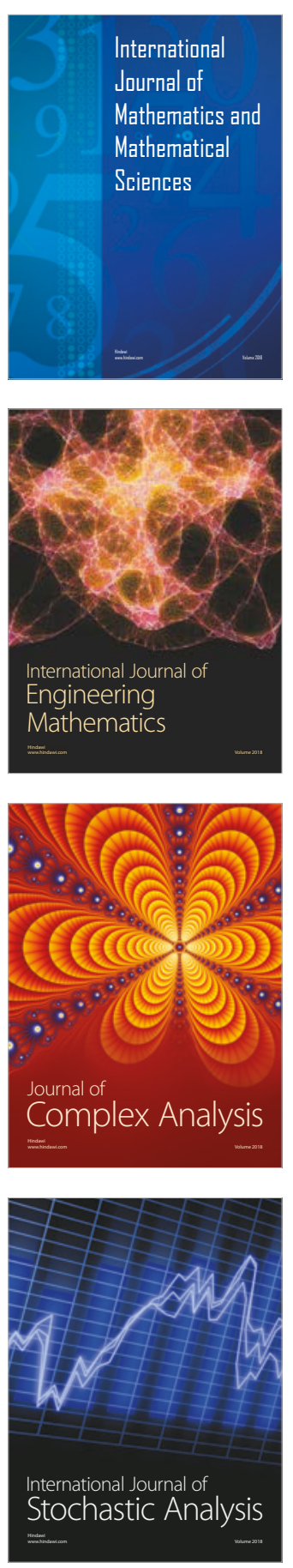
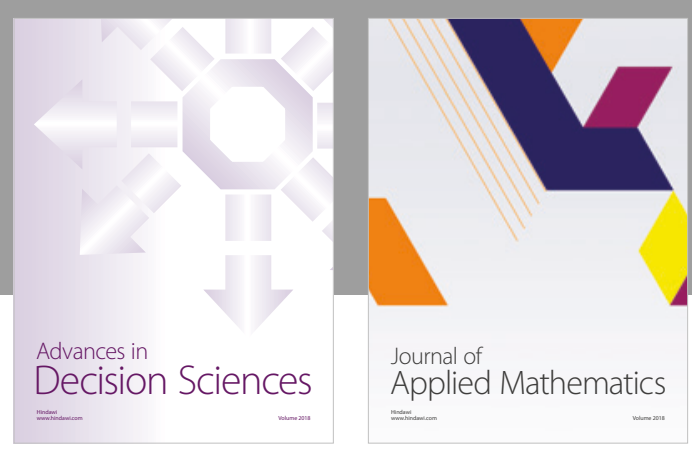

Journal of

Applied Mathematics
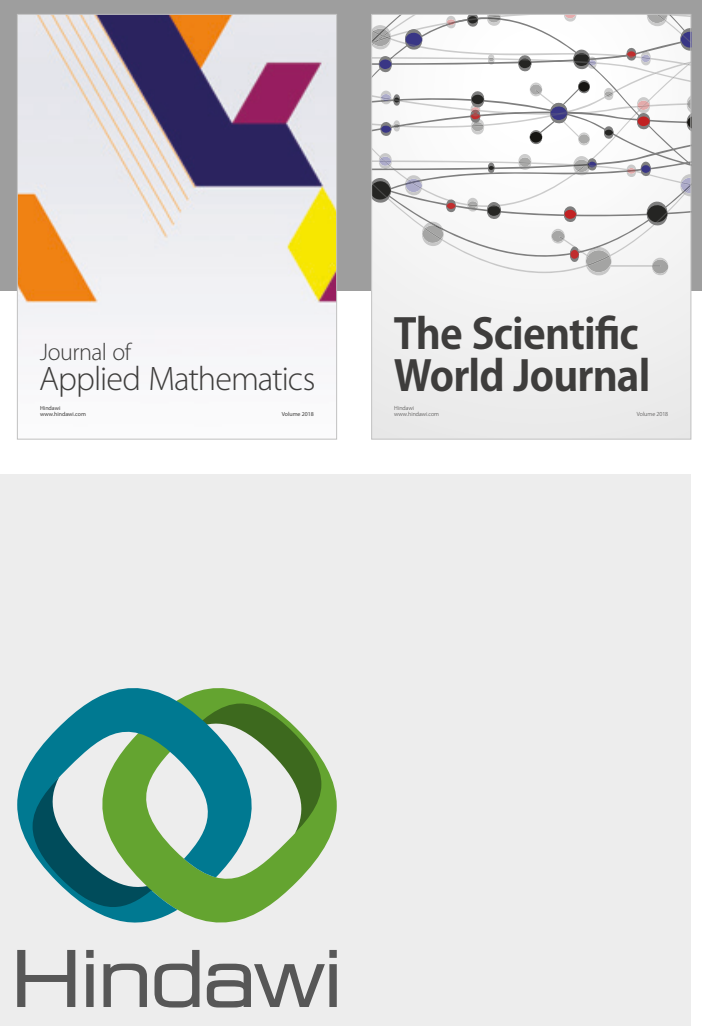

Submit your manuscripts at

www.hindawi.com

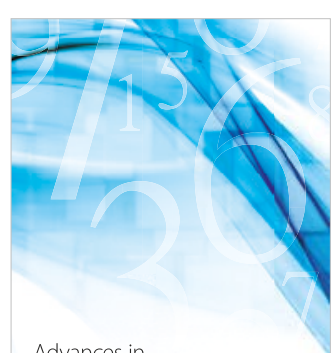

Advances in
Numerical Analysis
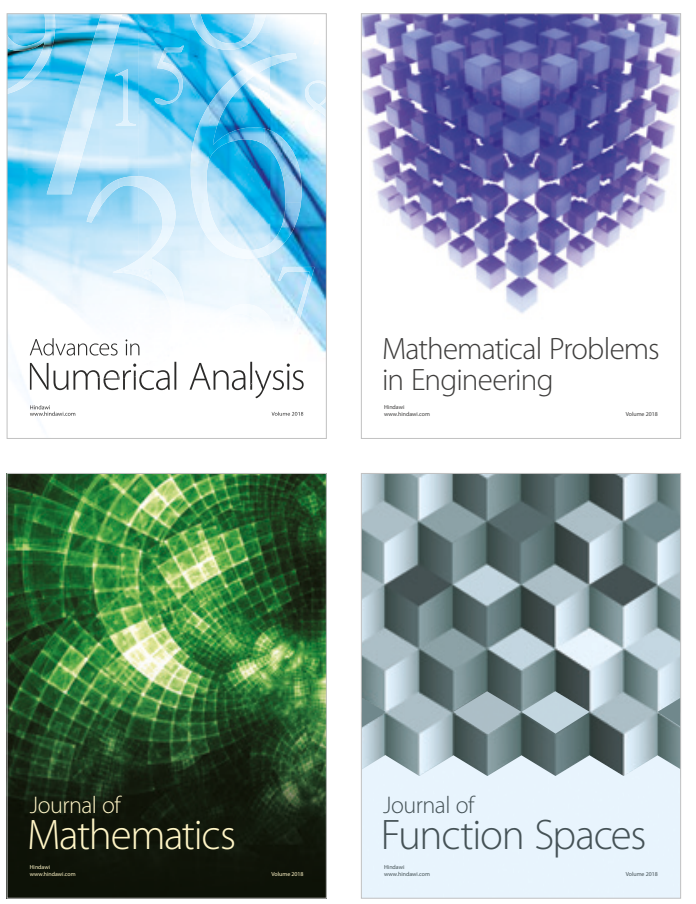

Mathematical Problems in Engineering

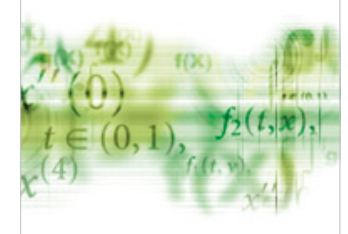

International Journal of

Differential Equations

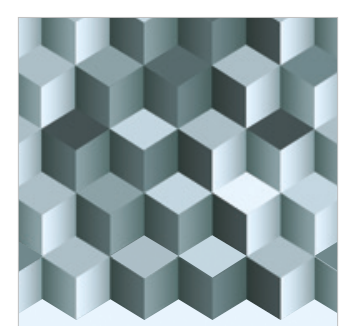

Journal of

Function Spaces

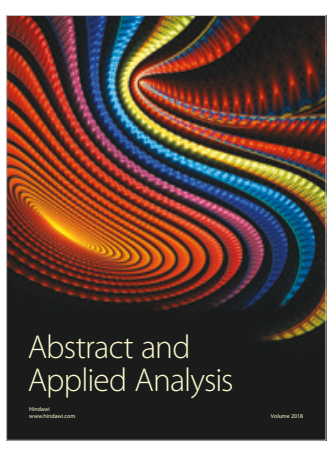

The Scientific

World Journal

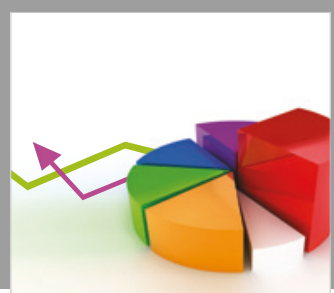

Journal of

Probability and Statistics
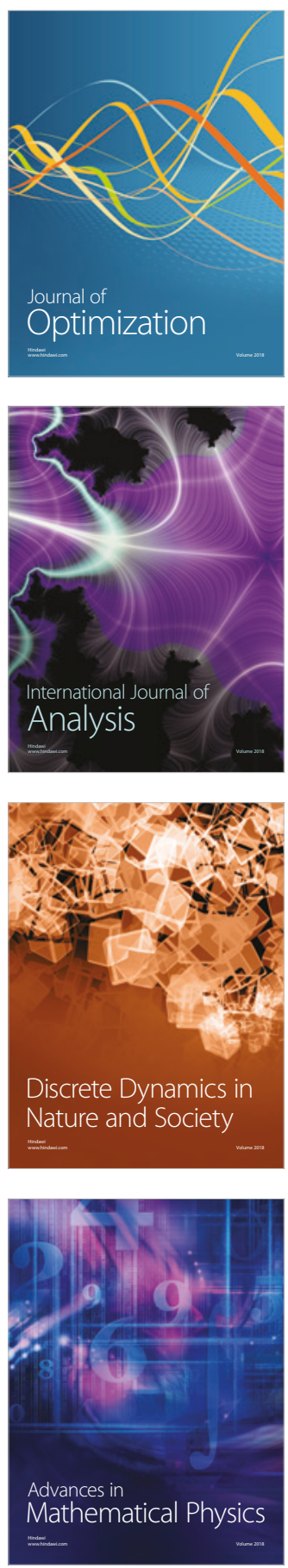